\title{
Periodic response of nonlinear dynamical system with large number of degrees of freedom
}

\author{
B P PATEL*, S M IBRAHIM and Y NATH \\ Department of Applied Mechanics, Indian Institute of Technology Delhi, \\ Hauz Khas, New Delhi 110 016, India \\ e-mail: badripatel@hotmail.com
}

MS received 5 February 2009; revised 5 May 2009

\begin{abstract}
In this paper, a methodology based on shooting technique and Newmark's time integration scheme is proposed for predicting the periodic responses of nonlinear systems directly from solution of second order equations of motion without transforming to double first order equations. The proposed methodology is quite suitable for systems with large number of degrees of freedom such as the banded system of equations from finite element discretization.
\end{abstract}

Keywords. Nonlinear; periodic response; shooting; time integration.

\section{Introduction}

Studies on the periodic responses of nonlinear dynamical systems are carried out mostly employing frequency domain or direct time domain integration methods. Both these methods are not sufficient to trace the unstable regions adequately. The harmonic balance or incremental harmonic balance methods lead to erroneous results if the correct number of harmonics is not included (Sundarajan \& Noah 1997). The direct time integration methods are computationally more involved for obtaining the steady state solutions of the large size weakly damped nonlinear systems and are unable to capture the unstable regions of the steady state response (Patel et al 2006, Ibrahim et al 2008). Finite element in time domain is also used for periodic solution of single degree of freedom linear systems (Gudla \& Ganguli 2006).

Periodic solutions of nonlinear systems can be obtained by shooting method. Unlike the frequency domain methods, the number of equations does not depend on the number of harmonics. Also, the steady state solutions can be reached in significantly less number of iterations as compared to the direct time integration methods. Another feature of the shooting method is that it yields the monodromy matrix, which can be used to predict the stability of solution (Nayfeh \& Balachandran 1994). For obtaining the periodic responses of second order systems using shooting method, the governing equations of motion are transformed into first order equations (Nayfeh \& Balachandran 1994; Ribeiro 2004; Ribeiro 2005; Ribeiro 2006; Padmanabhan \& Singh 1995). This transformation increases the computational time as the

*For correspondence 
number of equations is doubled and banded nature of system of equations is destroyed. This issue is quite important particularly for systems with large number of degrees of freedom such as the ones resulting from finite element discretization. To the best of the authors' knowledge, studies on the periodic solutions of nonlinear systems are limited to systems with few degrees of freedom in the existing literature. However, few degrees of freedom model of a continuous system may not capture a priori unknown modal interactions/phenomena.

Therefore, there is a need for the development of solution approach based on shooting technique which is capable of predicting periodic responses directly from solution of second order equations of motion and the present investigation is an effort in this direction. A methodology based on shooting method coupled with Newmark's implicit time integration technique is developed and its efficacy is demonstrated by considering circular cylindrical shell subjected to a discrete harmonic force for the first time in this paper.

\section{Solution methodology}

The discretized governing equations of motion at any time $t$ for systems with quadratic and cubic nonlinearities can be written as

$$
[\mathbf{M}][\ddot{\boldsymbol{\delta}}\}+[\mathbf{C}]\{\dot{\boldsymbol{\delta}}\}+\left[[\mathbf{K}]+\frac{1}{2}\left[\mathbf{K}_{\mathbf{1}}\{\boldsymbol{\delta}\}\right]+\frac{1}{3}\left[\mathbf{K}_{\mathbf{2}}\{\boldsymbol{\delta}\}\right]\right]\{\boldsymbol{\delta}\}=\{\mathbf{F}\},
$$

where $[\mathbf{M}]$ and $[\mathbf{C}]$ are mass and damping matrices; $[\mathbf{K}]$ is constant stiffness matrix; $\left[\mathbf{K}_{\mathbf{1}}(\boldsymbol{\delta})\right]$ and $\left[\mathbf{K}_{\mathbf{2}}(\boldsymbol{\delta})\right]$ are nonlinear stiffness matrices linearly and quadratically dependent on the displacement vector; $\{\ddot{\boldsymbol{\delta}}\},\{\dot{\boldsymbol{\delta}}\}$ and $\{\boldsymbol{\delta}\}$ are acceleration, velocity and displacement vectors; $\{\mathbf{F}\}$ is the force vector; and dot over the variables denotes derivative with respect to time.

For obtaining the periodic response of (1), the proposed methodology based on shooting technique, Newmark time integration scheme and Newton-Raphson iteration method is illustrated briefly.

In shooting method, state vector at time $t\left(=0\right.$ say) $\left\{\begin{array}{l}\boldsymbol{\delta}(0) \\ \dot{\boldsymbol{\delta}}(0)\end{array}\right\}=\{\boldsymbol{\eta}\}$ and solution $\left\{\begin{array}{l}\boldsymbol{\delta}\left(t, \eta ; \omega_{F}\right) \\ \dot{\delta}\left(t, \eta ; \omega_{F}\right)\end{array}\right\}$ with time period $T$ are sought such that

$$
\left\{\begin{array}{l}
\boldsymbol{\delta}\left(T, \eta ; \omega_{F}\right) \\
\dot{\boldsymbol{\delta}}\left(T, \eta ; \omega_{F}\right)
\end{array}\right\}_{2 N \times 1}=\{\boldsymbol{\eta}\}_{2 N \times 1} .
$$

where $\omega_{F}$ is the forcing frequency of harmonic excitation.

The value of time period $(T)$ is taken to be known for systems with harmonic excitation $\left(T=2 \pi / \omega_{F}\right)$ for response with fundamental and higher harmonics. For obtaining the branches of the periodic response curve with subharmonic participation, response time period $T$ is taken as integer multiple of fundamental period. For autonomous systems, time period $(T)$ is treated as an unknown and an additional equation such as phase condition/amplitude is required.

Since the state vector $\{\boldsymbol{\eta}\}$ is not known a priori, the solution is started from an initial guess $\left\{\boldsymbol{\eta}_{0}\right\}$ and correction $\{\Delta \eta\}$ is applied to this such that

$$
\left\{\begin{array}{l}
\boldsymbol{\delta}\left(T, \eta_{0}+\Delta \eta ; \omega_{F}\right) \\
\dot{\boldsymbol{\delta}}\left(T, \eta_{0}+\Delta \eta ; \omega_{F}\right)
\end{array}\right\}-\left\{\eta_{0}+\Delta \eta\right\} \approx\{\mathbf{0}\}
$$


Using Taylor series, (3) can be linearly approximated as:

$$
\left.\left[\begin{array}{l}
\frac{\partial \boldsymbol{\delta}}{\partial \eta}\left(T, \eta_{0} ; \omega_{F}\right) \\
\frac{\partial \dot{\boldsymbol{\delta}}}{\partial \eta}\left(T, \eta_{0} ; \omega_{F}\right)
\end{array}\right] \mathbf{I}\right]\{\Delta \eta\}=\left\{\eta_{0}\right\}-\left\{\begin{array}{l}
\boldsymbol{\delta}\left(T, \eta ; \omega_{F}\right) \\
\dot{\boldsymbol{\delta}}\left(T, \eta ; \omega_{F}\right)
\end{array}\right\},
$$

where $\frac{\partial \delta}{\partial \eta}$ and $\frac{\partial \dot{\boldsymbol{\delta}}}{\partial \eta}$ are $N \times 2 N$ matrices and $\mathbf{I}$ is $2 N \times 2 N$ identity matrix.

For the evaluation of $\frac{\partial \delta}{\partial \eta}$ and $\frac{\partial \dot{\delta}}{\partial \eta}$, differentiation of (1) with respect to $\eta$ gives

$$
[\mathbf{M}]\left[\frac{\partial \ddot{\boldsymbol{\delta}}}{\partial \boldsymbol{\eta}}\right]+[\mathbf{C}]\left[\frac{\partial \dot{\boldsymbol{\delta}}}{\partial \boldsymbol{\eta}}\right]+\left[\mathbf{K}_{T}\right]\left[\frac{\partial \boldsymbol{\delta}}{\partial \boldsymbol{\eta}}\right]=[\mathbf{0}],
$$

where $\left[\mathbf{K}_{T}\right]=[\mathbf{K}]+\left[\mathbf{K}_{1}(\delta)\right]+\left[\mathbf{K}_{2}(\delta)\right]$ is the tangent stiffness matrix.

The initial conditions associated with $(5)$ are:

$$
\left[\begin{array}{l}
\frac{\partial \delta}{\partial \eta} \\
\frac{\partial \delta}{\partial \eta}
\end{array}\right]_{t=0}=[\mathbf{I}]
$$

With marching in time, the solution of (1) starting from initial state vector $\left\{\boldsymbol{\eta}_{0}\right\}$ and that of (5) with initial condition given by (6) are obtained at any time $t$ using Newmark's time integration approach.

For the solution of (5) using Newmark's time integration approach, the quantities $\left[\frac{\partial \ddot{\delta}}{\partial \eta}\right]_{t+\Delta t}$ and $\left[\frac{\partial \dot{\delta}}{\partial \eta}\right]_{t+\Delta t}$ at time $t+\Delta t$ are written as:

$$
\begin{aligned}
& {\left[\frac{\partial \ddot{\boldsymbol{\delta}}}{\partial \boldsymbol{\eta}}\right]_{t+\Delta t}=a_{0}\left[\left[\frac{\partial \boldsymbol{\delta}}{\partial \boldsymbol{\eta}}\right]_{t+\Delta t}-\left[\frac{\partial \boldsymbol{\delta}}{\partial \boldsymbol{\eta}}\right]_{t}\right]-a_{2}\left[\frac{\partial \dot{\boldsymbol{\delta}}}{\partial \boldsymbol{\eta}}\right]_{t}-a_{3}\left[\frac{\partial \ddot{\boldsymbol{\delta}}}{\partial \boldsymbol{\eta}}\right]_{t}} \\
& {\left[\frac{\partial \dot{\boldsymbol{\delta}}}{\partial \boldsymbol{\eta}}\right]_{t+\Delta t}=a_{1}\left[\left[\frac{\partial \boldsymbol{\delta}}{\partial \boldsymbol{\eta}}\right]_{t+\Delta t}-\left[\frac{\partial \boldsymbol{\delta}}{\partial \boldsymbol{\eta}}\right]_{t}\right]-a_{4}\left[\frac{\partial \dot{\boldsymbol{\delta}}}{\partial \boldsymbol{\eta}}\right]_{t}-a_{5}\left[\frac{\partial \ddot{\boldsymbol{\delta}}}{\partial \boldsymbol{\eta}}\right]_{t}}
\end{aligned}
$$

where $a_{0}=\frac{1}{\beta \Delta t^{2}} ; a_{1}=\frac{\alpha}{\beta \Delta t} ; a_{2}=\frac{1}{\beta \Delta t} ; a_{3}=\frac{1}{2 \beta}-1 ; a_{4}=\frac{\alpha}{\beta}-1 ; a_{5}=\frac{\Delta t}{2}\left[\frac{\alpha}{\beta}-2\right] ; \alpha=$ $0.5 ; \beta=0.25$.

Time increment $\Delta t$ is chosen based on the convergence study.

Using (7) and (8), (5) becomes:

$$
\begin{gathered}
{\left[a_{0}[\mathbf{M}]+a_{1}[\mathbf{C}]+\left[\mathbf{K}_{T}\right]\right]\left[\frac{\partial \boldsymbol{\delta}}{\partial \boldsymbol{\eta}}\right]_{t+\Delta t}=\left[a_{0}[\mathbf{M}]+a_{1}[\mathbf{C}]\right]\left[\frac{\partial \boldsymbol{\delta}}{\partial \boldsymbol{\eta}}\right]_{t}} \\
+\left[a_{2}[\mathbf{M}]+a_{4}[\mathbf{C}]\right]\left[\frac{\partial \dot{\boldsymbol{\delta}}}{\partial \boldsymbol{\eta}}\right]_{t}+\left[a_{3}[\mathbf{M}]+a_{5}[\mathbf{C}]\right]\left[\frac{\partial \ddot{\boldsymbol{\delta}}}{\partial \boldsymbol{\eta}}\right]_{t}
\end{gathered}
$$

First, (9) is solved for $\left[\frac{\partial \delta}{\partial \eta}\right]_{t+\Delta t}$; then $\left[\frac{\partial \ddot{\delta}}{\partial \eta}\right]_{t+\Delta t}$ and $\left[\frac{\partial \dot{\delta}}{\partial \eta}\right]_{t+\Delta t}$ are obtained from (7) and (8). Using the solution of (5) at time period $T$, the correction of initial state vector $\{\Delta \eta)$ is obtained from (4). Again, (1), (5) and (4) are solved. This procedure is repeated until the state vector converges within the tolerance limit of $0.001 \%$. The simultaneous equations are 


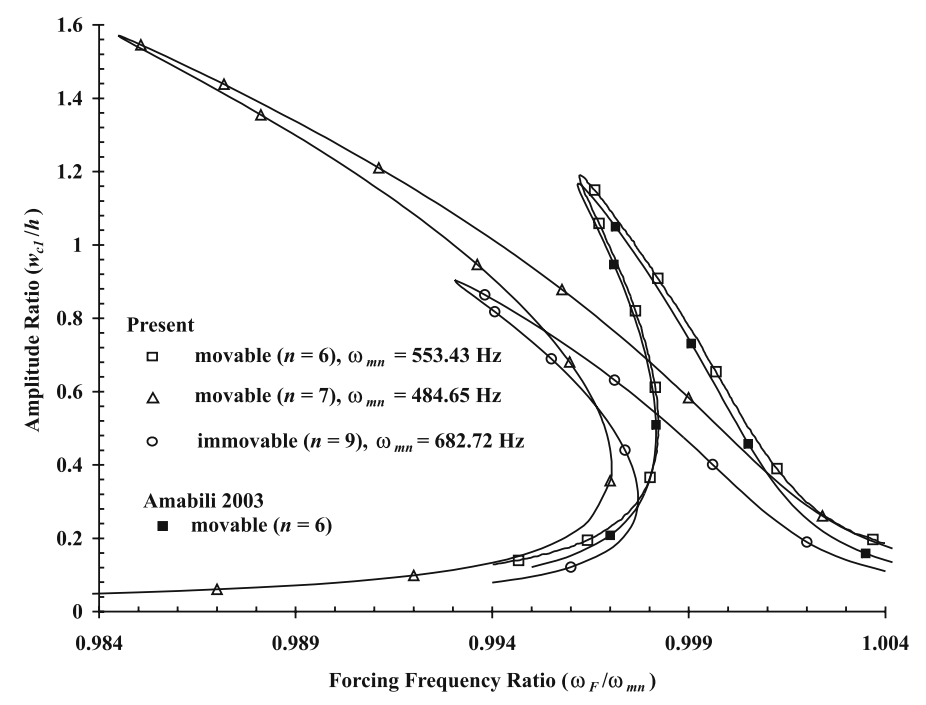

Figure 1. Steady state response versus forcing frequency curves for simply supported cylindrical shell.

solved using Choleskey factorization in the implementation of the approach by the authors. It can be noted that Choleskey factored left hand side of (9) is already available while solving (1). Additional computational cost is due to matrix multiplication on the right hand side and forward/back substitution which is quite straight forward for parallelization.

For obtaining the complete forcing frequency versus response amplitude curve, the analysis is carried out in two phases: (i) first starting from far enough from resonance (where periodic response is found to exist), forcing frequency is incremented and the periodic solutions are obtained and (ii) when the bifurcation points (identified by the eigenvalues of monodromy matrix) are encountered, the solutions are continued with the arc length continuation method. For the second phase, forcing frequency is treated as an unknown and incremental arc length is specified.

\section{Application example}

To demonstrate the application of the approach presented, simply supported circular cylindrical shell studied by Amabili (2003) is considered. The spatial solution is based on semianalytical finite element approach (Patel et al 2006). Dimensions and material properties of the shell considered are: length $(L)=0.2 \mathrm{~m}$, radius $\left(r_{o}\right)=0.1 \mathrm{~m}$, thickness $(h)=0.247 \mathrm{~mm}$, Young's modulus $(E)=71.02 \mathrm{GPa}$, density $(\rho)=2796 \mathrm{~kg} / \mathrm{m}^{3}$ and Poisson's ratio $(v)=$ $0 \cdot 31$. The shell is subjected to point harmonic force $F=F_{o} \operatorname{Sin}\left(\omega_{F} t\right)$ at the mid length of the shell with $F_{o}=0.0785 \mathrm{~N}$. The total number of degrees of freedom $(N)$ for the converged solution is 380 based on semi-analytical finite element discretization. The forcing frequency $\omega_{F}$ is varied in the neighbourhood of natural frequency $\omega_{m n}$ corresponding to mode with $m=1$ half waves in the length direction and $n$ full circumferential waves. The modal damping $\xi_{m n}=0.0005$ is assumed (Amabili 2003). The results are shown in figure 1 for movable $\left(v_{o}=w_{o}=\theta_{y}=0\right)$ and immovable $\left(u_{o}=v_{o}=w_{o}=\theta_{y}=0 ; u_{o}, v_{o}, w_{o}\right.$ are displacements of a point on the middle surface of the shell along axial, circumferential and 
thickness directions, and $\theta_{y}$ is rotation of the shell middle surface normal about axial direction) simply supported boundary conditions at the two ends of the shell. It can be seen from this figure that the steady state response amplitude - forcing frequency curve for movable simply supported shell is in good agreement with those of Amabili (2003) obtained employing AUTO 97 which deals with first-order equations. To further illustrate the approach, new results for movable $(n=7)$ and immovable $(n=9)$ simply-supported cases are also given in figure 1. The multi-valued response in the certain ranges of forcing frequency indicates the possibility of jump phenamena with increasing or decreasing forcing frequency in an experiment.

It is amply clear from this figure that the present approach is capable of tracing the full nonlinear response curve. Since it is based on time integration approach for the solution of second-order equations directly, it is quite suitable and can easily be incorporated in FEM based solutions of nonlinear systems with large number of degrees of freedom.

Financial support received from the Department of Science and Technology (DST), Govt. of India, New Delhi through Grant No. SR/S3/MERC/46/2005 is gratefully acknowledged.

\section{References}

Amabili M 2003 A comparison of shell theories for large-amplitude vibrations of circular cylindrical shells: Lagrangian approach. J. Sound and Vibration 264: 1091-1125

Gudla P K, Ganguli R 2006, Discontineous Galerkin finite element in time domain for solving periodic differential equations. Computer Methods in Applied Mechanics and Engineering 196: 682-696

Ibrahim S M, Patel B P, Nath Y 2008 On the nonlinear vibrations of clamped oval shells. Proceedings of the 9th Biennial ASME Conference on Engineering Systems Design and Analysis (ESDA 2008), July 7-9, 2008, Haifa, Israel

Nayfeh A H, Balachandaran B 1994 Applied Nonlinear Dynamics, (New York: John Wiley \& Sons)

Padmanabhan C, Singh R 1995 Analysis of periodically excited non-linear systems by a parametric continuation technique. J. Sound and Vibration 184: 35-58

Patel B P, Singh S, Nath Y 2006 Stability and nonlinear dynamic behaviour of cross-ply laminated heated cylindrical shells. Latin American Journal of Solids and Structures 3: 245-261

Ribeiro P 2004 Non-linear forced vibrations of thin/thick beams and plates by the finite element and shooting methods. Computers and Structures 82: 1413-1423

Ribeiro P 2005 Nonlinear vibrations of simply-supported plates by the $p$-version finite element method. Finite Elements in Analysis and Design 41: 911-924

Ribeiro P 2006 Forced periodic vibrations of laminated composite plates by a $p$-version, first order shear deformation finite element. Composite Science and Technol. 66: 1844-1856

Sundararajan P, Noah S T 1997 Dynamics of forced nonlinear systems using shooting/arc-length continuation method - Application to rotor systems. ASME J. Vibration and Acoustics 119: 9-20 